\title{
The compensation of patients injured in clinical trials
}

\author{
John M Barton, Maureen S Macmillan and Lindsay Sawyer Lothian Research Ethics Committee
}

\begin{abstract}
The problem of 'no fault' compensation for patients who suffer adverse effects as a result of their participation in clinical trials is discussed in the light of the guidelines issued by the Association of the British Pharmaceutical Industry ( $A B P I)$ and our recent experiences in reviewing protocols submitted to the local ethics of surgical research sub-committee. We have found a variety of qualifications being applied by pharmaceutical firms which are not in the spirit of the guidelines, let alone the interests of the patient, and we suggest a means whereby the patient can be assured of fair treatment in the event of 'no fault' injury.
\end{abstract}

\section{Introduction}

Over the past year or so, our local area ethics subcommittee has become concerned with the matter of compensation to patients who may be injured as a result of their taking part in a clinical trial; in particular, trials where a drug from a pharmaceutical company is being tested. Whilst we feel that in the overwhelming majority of cases, there is little if any risk of injury, it is of paramount importance that ethics committees ensure that the right of the patient to 'no fault' compensation be scrupulously safeguarded, a view shared by the BMA $(1,2)$, although not by everybody $(3,4)$. Our committee has spent considerable time both evolving and clarifying its ideas on the matter, and we believe that we have obtained a satisfactory form of wording both for the questions on the protocol which clinical researchers must submit, and for the notes of guidance which we now attach to the protocol. The notes of guidance $(5,6)$ seek to explain what is required by the local area ethics committee and suggest what we should find acceptable as a suitable response from the investigator and collaborating company. However, this amounts to ensuring that the patient is made fully aware of the risks and the arrangements, if any, for dealing with claims. The problem of multicentre trials where a company has obtained ethical

\section{Key words}

No fault compensation; ABPI guidelines; ethical approval. permission for the trial from other committees, who have not raised any objection to a restricted. compensation clause, has also exercised us. This $\omega$ multicentre problem has become still more complexक् in the context of the European Union (7). To ouro embarrassment a protocol was approved for an? earlier study using the same form of words which wec later found unacceptable. It is the purpose of this paper to share our experience with the widercommunity and to provide the means whereby $a_{\oplus}$ more uniform response can be achieved throughout. the UK, if not farther afield.

\section{Background}

The protocol form which is submitted to us by investigators contains the following questions:

\section{Question 1:}

In the event of a mishap, who will compensate the subjects? Will the pharmaceutical company compensate subjects or volunteers in the terms of the ABPI Guidelines (8)? In the event of death, damage or lasting disability being produced by a product involved in this study, who will compensateo the individual patient or volunteer?

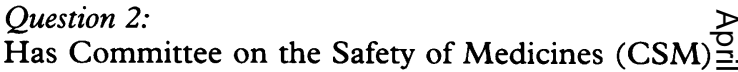
approval been given for all drugs to be used? ... If theN project is a drug trial sponsored or initiated by an industrial company, the company should provide aO written statement accepting strict liability for theirc products according to the ABPI guidelines, and $a$ copy of this should be submitted.

\section{Question 3:}

In the event of death, damage or lasting disability $\frac{7}{0}$ occurring as a result of negligence, who will accept responsibility for compensation?

The answer to question 3 is generally not a problem? and it is the restrictions which may be imposed by answers to questions 1 and 2 which we shall nowo explore further. The limitations which we have sought to exclude are detailed below. 


\section{Discussion}

In a report published by the Royal College of Physicians (9) on research involving patients it is observed that 'the risk of injury occurring as a result of participation in research is low but cannot be completely eliminated. In the present social climate there is no doubt that patients will expect that, should they be injured by participation in research, they will be compensated. Whatever the motives of the patient in agreeing to take part in research there is an element of benefit to society and it seems fair that society should bear some of the risk in financial terms. The patient's expectation that injury will be compensated is reasonable and we believe it has public support.'

Such injury can arise from three clearly identifiable causes:

1. Negligence of the clinician involved in administering the medicinal product.

2. Negligence on the part of the pharmaceutical company concerned, for example in failing to carry out the necessary minimum pre-trials research or in failing to specify certain exclusion criteria.

3. Other causes which are attributable to the medicinal product but in respect of which negligence cannot be established on the part of the clinician or the pharmaceutical company concerned.

The law as it stands - both in England and in Scotland - is that a patient is entitled to claim damages through the courts if negligence can be established under headings 1 or 2 above; but a patient has no entitlement to compensation in the United Kingdom if the situation falls under heading 3.

It is the duty of an ethical committee to ensure a patient can expect 'no fault' compensation in the event of injury attributable to a medicinal product used in the course of a clinical trial sponsored by a pharmaceutical company where only a Clinical Trial Certificate (CTC) or an Exemption Certificate (CTX) is held. This expectation does not arise where a product licence has been issued, and the product has been generally marketed in this country.

The spirit of 'no fault compensation' is set out in the Clinical Trial Compensation Guidelines (8), the 'basic principles' of which state the following:

1. Notwithstanding the absence of legal commitment, the company should pay compensation to patient-volunteers suffering bodily injury (including death) in accordance with these guidelines. 2. Compensation should be paid when, on the balance of probabilities, the injury was attributable to the administration of a medicinal product under trial or any clinical intervention or procedure provided for by the protocol that would not have occurred but for the inclusion of the patient in the trial.
The guidelines go on to set out, under the heading of Assessment of compensation:

'The amount of compensation paid should be appropriate to the nature, severity and persistence of the injury and should in general terms be consistent with the quantum of damages commonly awarded for similar injuries by an English court in cases where legal liability is admitted.'

Understandably the foregoing compensation may be abated in exceptional circumstances as where the patient is already suffering from a life-threatening disease. It is perhaps also worth noting that the Department of Health's advice to local research ethics committees differs from that provided by the Royal College of Physicians, the Medical Research Council and the Medicines Commission in the case of non-therapeutic research (10).

The European position is the same. In notes for guidance drawn up by the working party of the Committee for Proprietary Medicinal Products of the European Community (11) it is set out: 'In submitting clinical trials proposals to an ethics committee, they should be asked to consider the following ... (1.6e) Provision for compensation/ treatment in the case of injury or death of a subject if attributable to a clinical trial, and any insurance or indemnity to cover the liability of the investigator and sponsor.'

It should be added that the CPMP notes (11) were followed by a European Community directive (12). Part 4 of the annex to this directive, which is in substitution for the annex to directive $75 / 318 / \mathrm{EEC}$, sets out the relevant provisions for the particulars and documentation to accompany applications for marketing authorisation. Under the heading of Conduct of trials - good clinical practice, in section 1.1 it is directed that 'all phases of clinical investigation $\ldots$ be designed, implemented and reported in accordance with good clinical practice.'

The substitute annex is specifically referred to and adopted in the statutory instrument 2538 (13). Nevertheless, it is questionable whether the requirements in the directive confer on the patients any enforceable right in relation to compensation.

Thus, both in the UK and in Europe, there is an expectation that satisfactory provision is made for compensation in the event of any injury arising from a clinical trial. It is the nature of this 'satisfactory provision' that concerns us here.

In a number of recent protocols, where only a CTC or a CTX is held for the medicinal product, the pharmaceutical companies concerned have sought to limit the liability to the patient in the event of injury in a number of ways:

(a) Placing a maximum amount which may be claimed in relation to any one patient.

(b) Placing a limit on the aggregate which might be 
claimed by all patients in relation to the particular trial. On those occasions, it is not clear whether the limitation applies to the aggregate of world-wide claims or the claims attributable to research in one specific geographical area.

(c) Requiring claims to be intimated within three years of the date of the trial. This is significantly more restrictive than the normal limitation in negligence cases where the three-year period runs from the date of ascertainment of the injury.

(d) In the event of adverse circumstances, requiring the patient to consult initially the clinician involved in the trial. This might be impractical if the patient is temporarily abroad, or indeed, if the onset of injury is significantly delayed.

(e) In some cases, possible restrictions appear to be being placed upon the investigator over his or her right to publish, especially adverse, findings during the trial.

In correspondence, the pharmaceutical companies have offered to relax such limitations but this appears to be discretionary on the part of the companies. The last restriction, whilst not of direct concern to a participant, could lead to further patients or volunteers being put at unnecessary risk.

In certain instances, the pharmaceutical companies have insisted on such limitations whilst at the same time claiming that they were adhering to the ABPI guidelines. Such a stance involves reading qualifications into the ABPI guidelines which do not exist. The possibility of such varied interpretation has been discussed (14) and indeed the ABPI response to this commentary states that the views are not those of the ABPI and that the interpretation of the guidelines must remain a matter for the individual companies (15). Garden (16) gives the pragmatic response of one ethics committee to this as, for the time being, 'the interests of patients participating in research projects with new drugs would be adequately safeguarded if the company sponsoring the study agreed in writing to follow the ABPI guidelines as regards compensation for injury'.

Superficially, it appears that the pharmaceutical company is adhering to the guidelines but because of the specific limitations, the company could refuse full 'no fault' compensation in a particular case thereby depriving the patient of a full claim. This is clearly contrary to the public interest and public expectation.

We feel that where a pharmaceutical company seeks to adopt the ABPI Guidelines, these guidelines should be put forward without qualification and, for the avoidance of doubt, the willingness to accept liability should be set out in full. The latter is particularly important because pharmaceutical companies' interpretation of the guidelines is sometimes very loose, since presumably, the guidelines are, as their name implies, merely guidance notes. Further, any limitation as to liability under each or any of the headings (a) to (d) above should be? regarded as unacceptable. It is significant that in the $\overrightarrow{\bar{F}}$ guidelines published by the Royal College of Physicians of London (9) it is observed that 'some foreign companies and their UK-based subsidiaries $\overline{\bar{c}}$. make objections to accepting ABPI compensation $\widetilde{\Phi}$ guidelines even where the UK subsidiary is a member of the ABPI. The problem can normally be overcome by firm persistence.'

\section{Unlimited liability}

In the United Kingdom, there is unlimited liability in respect of damages arising from negligence whether in relation to a motor accident, an injury at $\omega$ work or a claim against a health authority; and there $\vec{D}$ is no reason why the liability of pharmaceutical ${ }_{\circ}^{\circ}$ companies in relation to 'no fault' compensation ${ }^{\text {? }}$ claims should not be the same.

In other situations, pharmaceutical companies have expressed willingness to: (i) indemnify the ${ }_{-}^{\mathbb{\Phi}}$ clinician, (ii) only pay compensation in excess of the $\vec{\theta}$ limit provided by the clinician's insurers, or (iii) provide insurance direct to the patient.

None of these proposals is acceptable. A乏े clinician is only liable in respect of his or her own negligence. This is ordinarily covered by the clinician's own medical insurer and therefore an $\frac{\Phi}{\varnothing}$ offer to indemnify the clinician is of no benefit to $\Rightarrow$ the patient in the event of injury. So far as (ii) is윽 concerned, this is merely an unnecessary? complication, for as stated above, it is only in respect of claims resulting from the clinician's own negligence that will fall to be dealt 'with by his insurers. In regard to (iii) it may be informative foro the patient to know that any claim for injury will be. entertained by the pharmaceutical company's owno insurers but the liability should initially lie directly with the pharmaceutical company - as is the case in? any other civil claim - because the patient will have no knowledge of the 'small print' in the insurance을. cover which is being offered by the company. It isn interesting to note that a number of companies, when asked about their plans for compensation give 0 full and fulsome details of the plans for covering the $N$ investigator, whilst not mentioning the patient at all.

Finally it should be pointed out that if 'no fault' liability is not being accepted by the pharmaceutical ${ }^{?}$ company concerned, and if this is acceptable to the ethics committee in the exceptional circumstances of the particular research, then such limited liability should be clearly set out in the information sheet provided to the patient so that the patient will be fully aware that he or she cannot expecto straightforward compensation in the event of seriouso injury attributable to the particular medicinal product under trial. 


\section{Conclusions}

The answers to questions 1 and 2 posed at the outset, which we require are 1) that the company will pay compensation according to the ABPI Guidelines on a no fault basis and 2) that a suitable certificate has been obtained (or has been applied for - in this event, the start of the trial must await its availability). Together with these answers, a statement from the company indicating that it does indeed accept the basis of no fault compensation must be produced, and, recently, our health board has also required that evidence be produced that the company is able to fulfil this responsibility. The short statement below which is based upon a memorandum from the region's ethics of medical research committee is now routinely sent out with our protocol forms, since it provides a concise and satisfactory statement of what we feel are the minimum requirements for safeguarding the patient's right to compensation. Only after satisfactory statements to this effect are obtained will ethical approval be given. We do not feel that there is anything unreasonably open-ended about these requirements, since they are those already agreed by the pharmaceutical industry itself.

The answer to question 1 should include a clear statement regarding 'no fault' compensation. The following is the suggested guideline for drug companies involved:

We confirm that, in principle, our company accepts liability to the patient on a 'no fault' compensation basis which means that the patient will receive compensation for injury regardless of whether he or she is able to prove that our company has been negligent in relation to research or development of the medicinal product under trial or that the product is defective.

We also confirm that the amount of any compensation to be paid will be appropriate to the nature, severity and persistence of any injury which was reasonably caused by the materials and/or substances applied in the clinical trial and/or the activities performed with the patient's body in connection with the trial and will in general terms be consistent with the quantum of damages commonly awarded for similar injuries by an English court in cases where legal liability is admitted. We further confirm that our company has insurance cover for such claims.

Recently, the first paragraph of the above has been found controversial by some companies with experience of US legislation and a possible solution in such cases is to replace it by section 1.7 of (8):

We confirm that our company will pay compensation regardless of whether the patient is able to prove that the company has been negligent in relation to research or development of the medicinal product under trial or that the product is defective and therefore, as the producer, the company is subject to strict liability in respect of the injuries caused by it.

\section{Acknowledgements}

We are grateful to several companies and one referee for their forbearance and help during the evolution of the ideas expressed in this paper. We are also grateful to the Lothian Health Board for their help and for permission to reproduce parts of a memorandum.

Fohn M Barton, $M A, L L B$, is a Solicitor. Maureen $S$ Macmillan, $B A, P h D, R C N$, is Research Officer, Borders Health Board, Edinburgh University. Lindsay Sawyer, BSc, PhD, is Reader in the Department of Biochemistry, Edinburgh University.

\section{References}

(1) British Medical Association. No fault compensation. London: BMA, 1987.

(2) Smith R. Reconsidering compensation for medical accidents. British medical journal 1992; 304: 1066-1067.

(3) Rosen M. Medical negligence. British medical journal 1992; 304: 576.

(4) Smith R. Fiddling with medical negligence. British medical journal 1992; 304: 198-199.

(5) Scottish Office Home and Health Department. Local research ethics committees. Edinburgh: SHDD, 1991.

(6) Department of Health. Local research ethics committees. London: Department of Health, 1991 (ref HSG [91]5).

(7) Riis P. Medical ethics in the European Community. fournal of medical ethics 1993; 19: 7-12.

(8) Association of the British Pharmaceutical Industry. Clinical trial compensation guidelines. London: ABPI, 1991. These guidelines are not materially different from those set out in 1983, but still used by some companies.

(9) Royal College of Physicians of London. Guidelines on the practice of ethics committees in medical research involving human subjects [2nd ed]. London: RCP, 1990.

(10) Gillon R. No fault compensation for victims of nontherapeutic research - should government continue to be exempt? Fournal of medical ethics 1992; 18: 59-60.

(11) CPMP Working Party on Efficacy of Medicinal Products. EEC note for guidance: Good clinical practice for trials on medicinal products in the European Community. Brussels: EEC, 1990.

(12) Commission directive 1991 Jul 19: 91/507/EEC. Official journal of the European communities no L, 270/32-50.

(13) The medicines (applications for grant of product licences products for human use) regulations 1993. Statutory instruments 1993 No 2538.

(14) Diamond A L, Laurence D R. Compensation and drug trials. British medical journal 1983; 287: 675-677.

(15) Massam A D W. Compensation and drug trials. British medical journal 1983; 287: 1066.

(16) Garden M. Compensation and drug trials. British medical journal 1983; 287: 1381. 